\title{
Using Factorization to Estimate the Charmed Meson Decays
}

\author{
Nguyen Thu Huong ${ }^{*}$, Ha Huy Bang \\ Faculty of Physics, VNU University of Science, \\ 334 Nguyen Trai, Thanh Xuan, Hanoi, Vietnam \\ Received 15 September 2016 \\ Revised 28 September 2016; Accepted 30 September 2016
}

\begin{abstract}
Study of the charmed meson decays is mentioned in the articles [1, 2]. These researches help to improve the results of light mesons decays. In this paper, applying the factorization method, we try to estimate the branching ratios of charmed meson decays, namely $D_{(s)}^{+} \rightarrow \pi^{+} \eta\left(\rightarrow \mu^{+} \mu^{-}\right)$. This can be an effective method for computing the decay rates of new channels.

Keywords: Factorization, charmed meson decays, operator product expansion.
\end{abstract}

\section{Introduction}

Quantum Chromodynamics (QCD) is the theory of strong interaction we do not understand well at low energy. For the new channels, we would liketo look for the suitable approximation method to estimate the decay rates and cross-sections. One of those methods we would like to mention in the article is factorization.

Factorization in the case of semi - leptonic decays with short and long distance QCD are researched in some articles [3], not mentioned in our article. And the case of non - leptonic D-decays in which the final state consists exclusively out of hadrons is a completely different story. Here even the matrix elements entering the simplest decays, the two body decays like $D_{s}^{+} \rightarrow \pi^{+} \eta, D^{+} \rightarrow \bar{K}^{0} \pi^{+}$ cannot be calculated in QCD reliably at present. For this reason approximative schemes for these decays can be found in the literature. One of such schemes, the factorization scheme for matrix elements has been popular for some time among experimentalists and phenomenologists.

Factorization is the effective approximation to estimate the amplitude of pseudo-scalar decays. The law of factorization is reducing the hadronic matrix elements of four-quark operators to products of current matrix elements.

In this article, we would like to give some brief introduction to Operator Production Expansion and Factorization, and Section 3 gives some applications to deduce the decay rates and applying this method to the new channels in the future.

\footnotetext{
${ }^{*}$ Corresponding author. Tel.: 84-988768887

Email: huong.nguyenthu@ vnu.edu.vn
} 


\section{Operator Product Expansion (OPE) and Factorization}

\subsection{Operator Product Expansion}

We introduce briefly to OPE.

The basic idea of OPE [3]: the product of two charged current operators is expanded into a series of local operators, whose contributions are weighted by effective coupling constants, the Wilson coefficient.

Due to the asymptotic freedom of QCD, the short distance QCD corrections to weak decays, that is the contribution of hard gluon at energies of the order $O\left(M_{W}\right)$ down to hadronic scales $O(1 \mathrm{GeV})$. Taking one simple example of the $c \rightarrow s u \bar{d}$ transition,

Without QCD effects:

$$
H_{e f f}^{(0)}=\frac{G_{F}}{\sqrt{2}} V_{c S}^{*} V_{u d}\left(\bar{s}_{\alpha} c_{\alpha}\right)_{V-A}\left(\bar{u}_{\beta} d_{\beta}\right)_{V-A}
$$

With QCD effects after integrating out the heavy W-boson and top-quark fields,

$$
H_{e f f}^{(0)}=\frac{G_{F}}{\sqrt{2}} V_{c S}^{*} V_{u d}\left(c_{1}(\mu) Q_{1}+c_{2}(\mu) Q_{2}\right)
$$

Where

$$
\begin{aligned}
& Q_{1}=\left(\bar{s}_{\alpha} c_{\beta}\right)_{V-A}\left(\bar{u}_{\beta} d_{\alpha}\right)_{V-A} \\
& Q_{2}=\left(\bar{s}_{\alpha} c_{\alpha}\right)_{V-A}\left(\bar{u}_{\beta} d_{\beta}\right)_{V-A}
\end{aligned}
$$

The essential features of this Hamiltonian are:

- Beside the original $Q_{2}$, there has a new operator $Q_{1}$ with the same flavor form but different colour structure is generated. They contain the product of the colour charge $T_{\alpha \beta}^{a}, T_{\gamma \rho}^{a}$ following colour algebra:

$$
T_{\alpha \beta}^{a} T_{\gamma \rho}^{a}=-\frac{1}{2 N} \delta_{\alpha \beta} \delta_{\gamma \rho}+\frac{1}{2} \delta_{\alpha \rho} \delta_{\gamma \beta}
$$

- The first term in the r.h.s is a correction to the coefficient of the operator $Q_{2}$ and the second term in the r.h.s is the value to the new operator $Q_{1}$.

$c_{1}, c_{2}$ coupling constant for the interaction term $Q_{1}, Q_{2}$ become calculable non-trivial function of $\alpha_{s}, M_{W}$ and the renormalization scale $\mu$.

The purpose is calculation $c_{1}, c_{2}$ in the ordinary perturbation theory. $c_{1}, c_{2}$ can be determined by the requirement that the amplitude $A_{\text {full }}$ in the full theory be reproduced by the corresponding amplitude in the effective theory:

$$
A_{\text {full }}=A_{\text {eff }}=\frac{G_{F}}{\sqrt{2}} V_{c s}^{*} V_{u d}\left(c_{1}(\mu)<Q_{1}>+c_{2}(\mu)<Q_{2}>\right)
$$

This method is called "the matching of the full theory onto the effective theory".

The matching procedure gives the values:

$$
c_{1}(\mu)=-3 \frac{\alpha_{s}}{4 \pi} \ln \frac{M_{W}^{2}}{\mu^{2}} ; c_{2}(\mu)=1+\frac{3}{N} \frac{\alpha_{s}}{4 \pi} \ln \frac{M_{W}^{2}}{\mu^{2}}
$$

where $\mathrm{M}_{\mathrm{W}}$ is the mass of $\mathrm{W}$ boson. 
When considering from $\mathrm{M}_{\mathrm{W}}$ down to the scale $\mu$, we have to sum the large logarithms to all order of perturbation theory[3]. We can write the combination $c_{ \pm}(\mu)=c_{1}(\mu) \pm c_{2}(\mu)$ of the Wilson coefficients under change of the renormalization scale. They can be obtained from the solution of the RGE $[4,5]$,

$$
\left(\mu \frac{d}{d \mu}-\Gamma_{ \pm}\right) c_{ \pm}(\mu)=0
$$

with the initial condition $c_{ \pm}\left(m_{W}\right)=1$, and then $c_{1}\left(m_{W}\right)=1, c_{0}\left(m_{W}\right)=0$.

At one-loop order, they are given by $\Gamma_{ \pm}=\gamma_{ \pm} \frac{\alpha_{s}}{4 \pi} ; \gamma_{ \pm}=6\left( \pm 1-\frac{1}{N_{C}}\right)$ where $\mathrm{N}_{\mathrm{C}}=3$ is the number of the colors. To leading logarithmic order (LO), the solution of the RGE is

$$
c_{ \pm}(\mu)=\left(\frac{\alpha_{s}\left(m_{W}\right)}{\alpha_{s}(\mu)}\right)^{\gamma_{ \pm} / 2 \beta_{0}} ; \quad \beta_{0}=\frac{11}{3} N_{C}-\frac{2}{3} n_{f}
$$

Where $\beta_{0}$ is the first coefficient of the $\beta$ function, and $\mathrm{n}_{\mathrm{f}}$ is the number of active flavors (in the region between $m_{W}$ and $\mu$ ).

\subsection{Factorization}

By factorizing the matrix elements of the four quark operators contained in the effective Hamiltonian, there are three classes of decays [6].

Class (I): Only a charged meson can be generated directly from a color - singlet current, for typical example: $D_{s}^{+} \rightarrow \pi^{+} \eta$ (Figure 1)

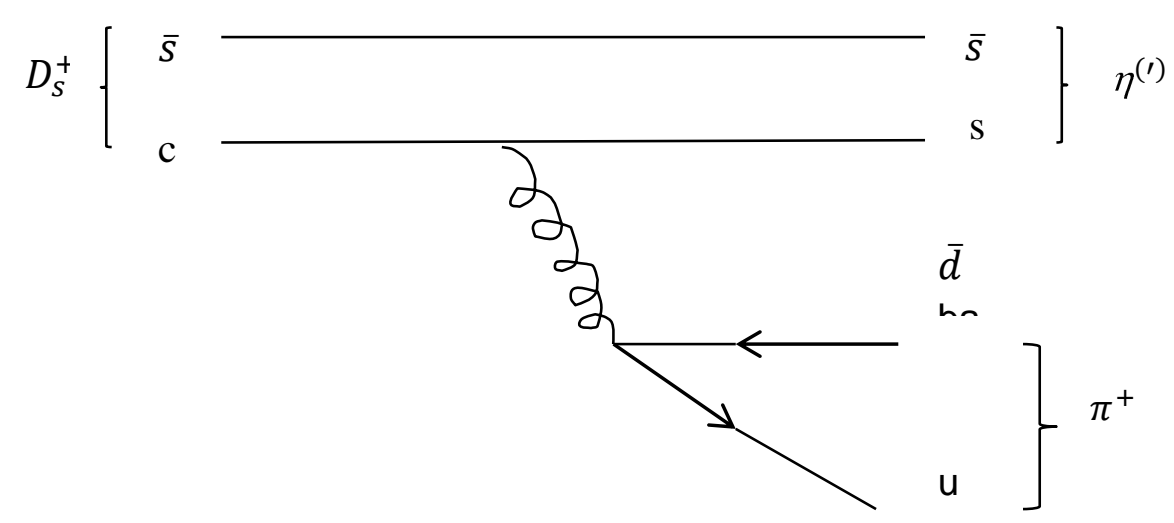

Figure 1. Typical diagram of $D_{S}^{+} \rightarrow \pi^{+} \eta^{(\prime)}$ for Class I

For these processes, the relevant QCD coefficient is given by the combination:

$$
a_{1}=c_{1}\left(\mu_{f}\right)+\xi c_{2}\left(\mu_{f}\right) \quad(\text { Class } I)
$$

Where $\xi=1 / N_{C}\left(\mathrm{~N}_{C}\right.$ being the number of quark colors $)$, and $\mu_{f}=O\left(m_{b}\right)$ is the scale at which factorization is assumed to be relevant.

Class (II): consists of those decays where the meson generated directly from the current is neutral like the particle in the decay as Figure 2 


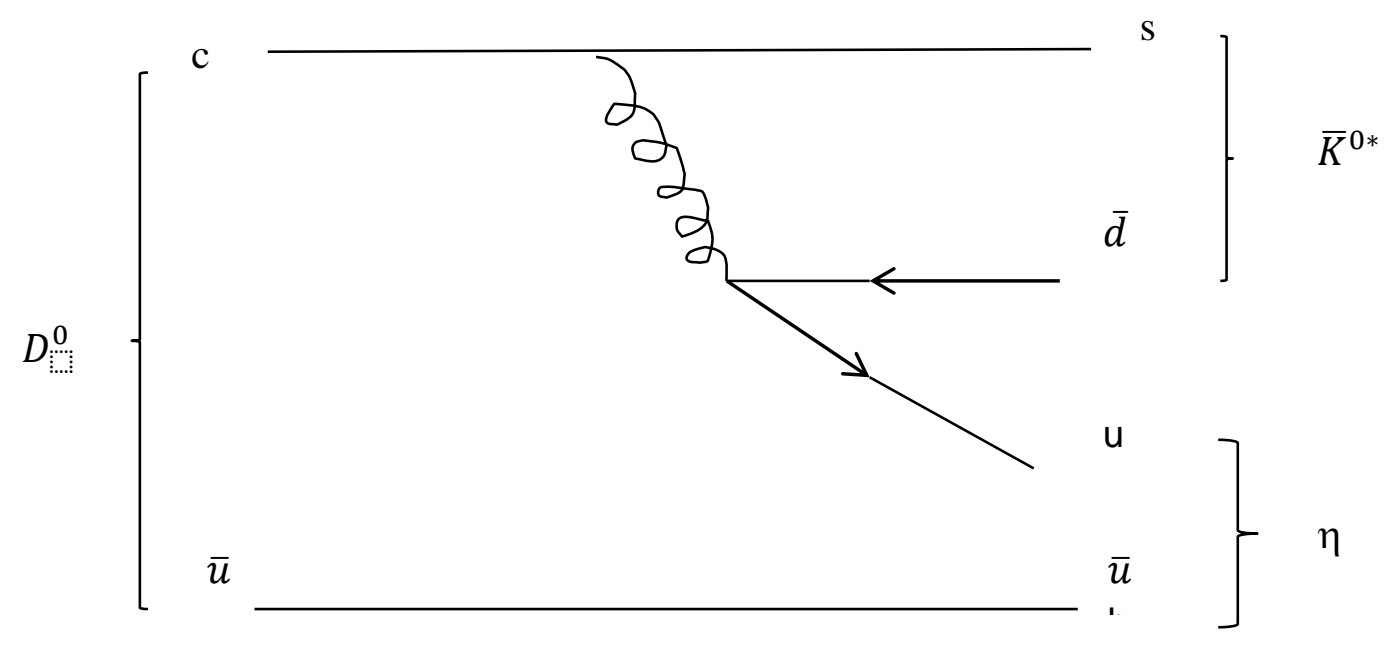

Figure 2. Typical diagram of for Class II.
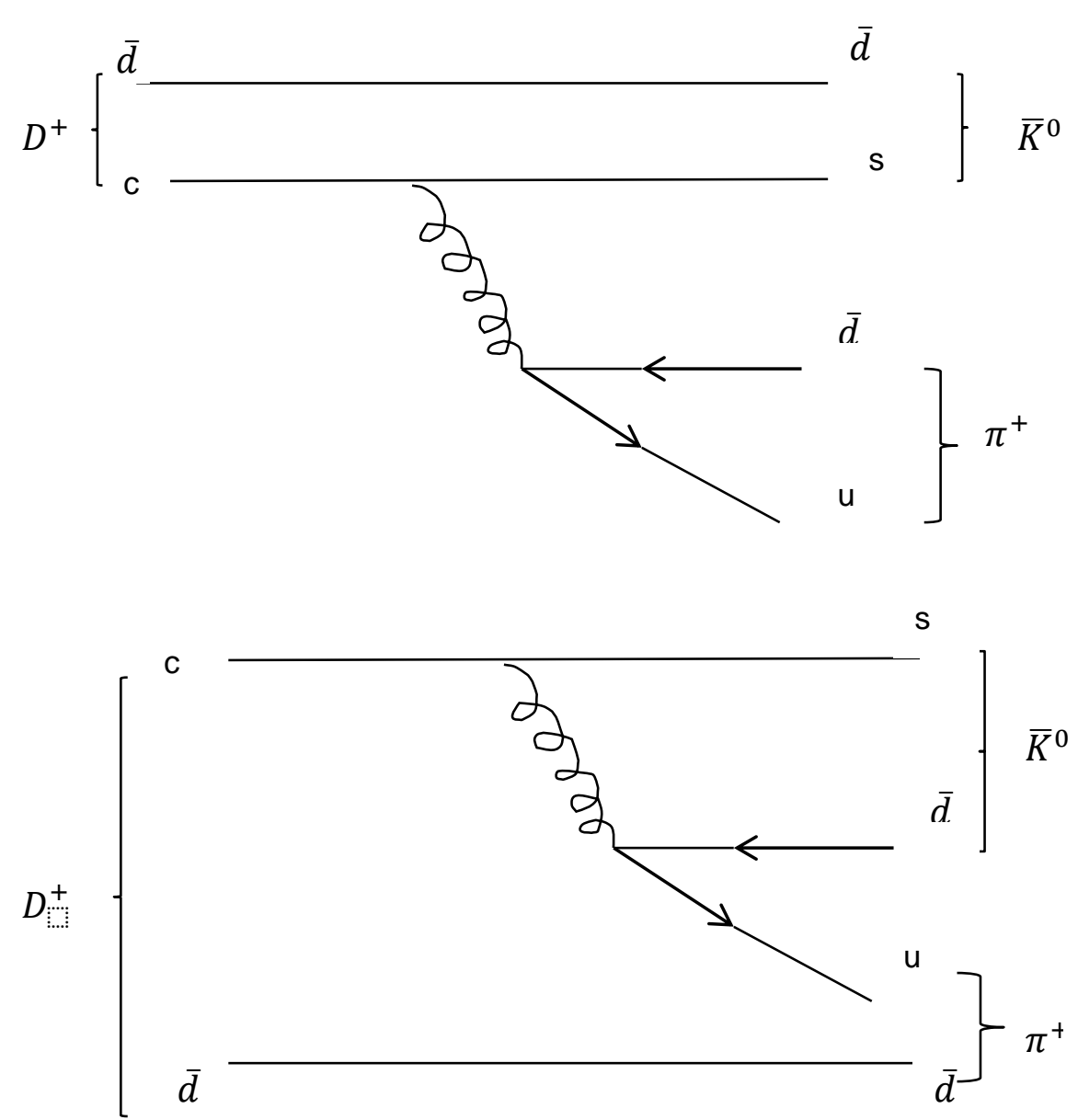

Figure 3. Typical diagram of $D^{+} \rightarrow \bar{K}^{0} \pi^{+}$for Class III. 
The decay amplitude

$$
A_{\text {fact }}=\frac{G_{F}}{\sqrt{2}} V_{u d} V_{c S}\left\langle\bar{K}^{0 *}\left|A_{\mu}\right| 0\right\rangle\left\langle\eta\left|V_{\mu}\right| D^{0}\right\rangle
$$

Where QCD coefficient: $a_{2}=c_{2}\left(\mu_{f}\right)+\xi c_{1}\left(\mu_{f}\right) \quad$ (Class II)

Class (III): The decays which the final state contains a charged meson and a neutral meson, which means $\mathrm{a}_{1}, \mathrm{a}_{2}$ amplitude interfere, such as $D^{+} \rightarrow \bar{K}^{0} \pi^{+}$(Figure 3)

The corresponding amplitudes involve a combination $\mathrm{a}_{1}+\mathrm{xa}_{2}$ (Class III) where $\mathrm{x}=1$ in the formal limit of a flavor symmetry for the final-state mesons.

\section{Applications for the $D$ meson decays into $\eta\left({ }^{\circ}\right)$ meson}

From PDG [7], we have an equation as below:

$$
\left\{\begin{array}{l}
\eta=\cos \theta_{P} \eta_{8}-\sin \theta_{P} \eta_{0} \\
\eta^{\prime}=\sin \theta_{P} \eta_{8}+\cos \theta_{P} \eta_{0}
\end{array}\right.
$$

where $\mathrm{SU}(3)$-octet and -singlet states are:

Using this condition $[8,9] \theta_{\mathrm{P}}=\alpha_{\mathrm{P}}+\theta_{\mathrm{I}}-\pi / 2$ in which: $\theta_{I}=\tan ^{-1}\left(\frac{1}{\sqrt{2}}\right) ; \alpha_{P}=\sin ^{-1}\left(\frac{1}{\sqrt{3}}\right)$, we obtain:

\subsection{Calculation of the branching ratio $\boldsymbol{D}_{\boldsymbol{s}}^{+} \rightarrow \boldsymbol{\pi}^{+} \boldsymbol{\eta}^{(\prime)}$}

From Figure 1, we calculate the amplitude (see Appendix)

$$
\begin{aligned}
& A\left(D_{s}^{+} \rightarrow \pi^{+} \eta^{\prime}\right)=\frac{2}{\sqrt{6}} T^{\prime} \\
&= \frac{2}{\sqrt{6}} a_{1}\left\langle\eta^{\prime}\left|V_{\mu}\right| D_{s}^{+}\right\rangle\left\langle\pi^{+}\left|A_{\mu}\right| 0\right\rangle \sim \frac{2}{\sqrt{6}} a_{1} f_{\pi}\left(m_{D}^{2}\right. \\
&\left.-m_{\eta^{\prime}}^{2}\right) F_{0}^{D_{s}^{+} \rightarrow \eta^{\prime}}(0) \\
& A\left(D_{s}^{+} \rightarrow \pi^{+} \eta\right)=\frac{1}{\sqrt{3}} T=\frac{1}{\sqrt{3}} a_{1}\left\langle\eta\left|V_{\mu}\right| D_{s}^{+}\right\rangle\left\langle\pi^{+}\left|A_{\mu}\right| 0\right\rangle \sim \frac{1}{\sqrt{3}} a_{1} f_{\pi}\left(m_{D}^{2}-m_{\eta}^{2}\right) F_{0}^{D_{s}^{+} \rightarrow \eta}
\end{aligned}
$$

Making the assumption $F_{0}^{D_{s}^{+} \rightarrow \eta^{\prime}}(0)=F_{0}^{D_{s}^{+} \rightarrow \eta}(0)$, we obtain

$$
B R\left(D_{S}^{+} \rightarrow \pi^{+} \eta^{\prime}\right)=2 \frac{\left(m_{D}{ }^{2}-m_{\eta^{\prime}}^{2}\right)^{2}}{\left(m_{D}{ }^{2}-m_{\eta}^{2}\right)^{2}} B R\left(D_{S}^{+} \rightarrow \pi^{+} \eta\right)
$$

We have a numerical calculation: $B R\left(D_{s}^{+} \rightarrow \pi^{+} \eta^{\prime}\right)=2.31 \%$. Compare with the experiment value from PDG [7], $B R_{\exp }\left(D_{s}^{+} \rightarrow \pi^{+} \eta^{\prime}\right)=(3.94 \pm 0.25) \%$, it can be an acceptable approximation.

\subsection{Calculation of the branching ratio $\boldsymbol{D}^{\mathbf{0}} \rightarrow \overline{\boldsymbol{K}}^{\mathbf{0} *} \boldsymbol{\eta}$}

Using the factorization method, the amplitude of decay (Figure 2) is obtained as the same way as $A\left(D_{S}^{+} \rightarrow \pi^{+} \eta^{\prime}\right)$

$$
\begin{aligned}
A\left(D^{0} \rightarrow \bar{K}^{0 *} \eta\right) & =-\frac{1}{\sqrt{3}} V_{c S} V_{u d} C_{s}=-\frac{1}{\sqrt{3}} C_{s}=-\frac{1}{\sqrt{3}} a_{2}\left\langle\bar{K}^{0 *}\left|A_{\mu}\right| 0\right\rangle\left\langle\eta\left|V_{\mu}\right| D^{0}\right\rangle \sim \\
& =-\frac{1}{\sqrt{3}} a_{2} f_{K}\left(m_{D^{0}}^{2}-m_{\eta}^{2}\right) F_{0}^{D^{0} \rightarrow \eta}(0)
\end{aligned}
$$


Similarly, the amplitude of $D^{0} \rightarrow \bar{K}^{0 *} \pi^{0}$ decay

$$
A\left(D^{0} \rightarrow \bar{K}^{0 *} \pi^{0}\right)=\frac{1}{\sqrt{2}} a_{2} f_{K}\left(m_{D^{0}}^{2}-m_{\pi^{0}}^{2}\right) F_{0}^{D^{0} \rightarrow \pi^{0}}(0)
$$

Now we get the ratio between two channels $D^{0} \rightarrow \bar{K}^{0 *} \eta, D^{0} \rightarrow \bar{K}^{0 *} \pi^{0}$

$$
\frac{B R\left(D^{0} \rightarrow \bar{K}^{0 *} \eta\right)}{B R\left(D^{0} \rightarrow \bar{K}^{0 *} \pi^{0}\right)}=\frac{\tau\left(D^{0}\right)\left|A\left(D^{0} \rightarrow \bar{K}^{0 *} \eta\right)\right|^{2}}{\tau\left(D^{0}\right)\left|A\left(D^{0} \rightarrow \bar{K}^{0 *} \pi^{0}\right)\right|^{2}}=\frac{2}{3}\left(\frac{\left(m_{D^{0}}^{2}-m_{\eta}^{2}\right) F_{0}^{D^{0} \rightarrow \eta}(0)}{\left(m_{D^{0}}^{2}-m_{\pi^{0}}^{2}\right) F_{0}^{D^{0} \rightarrow \pi^{0}}(0)}\right)^{2}
$$

Using the assumption $F_{0}^{D^{0} \rightarrow \eta}(0)=F_{0}^{D^{0} \rightarrow \pi^{0}}(0)=F_{0}^{D^{0} \rightarrow \pi^{-}}(0)=\frac{0.152}{V_{c d}}$, and the value from PDG, $B R\left(D^{0} \rightarrow \bar{K}^{0 *}(892)\left(\rightarrow K_{S}^{0} \pi^{0}\right) \pi^{0}\right)=(7.8 \pm 0.7) \times 10^{-3}, \quad$ we obtain $\quad B R\left(D^{0} \rightarrow \bar{K}^{0 *}(892)(\rightarrow\right.$ $\left.\left.K_{S}{ }^{0} \pi^{0}\right) \eta\right)=4.4 \times 10^{-3}$. Comparing with the experimental value [7], $B R\left(D^{0} \rightarrow \bar{K}^{0 *}(892)(\rightarrow\right.$ $\left.\left.K_{S}{ }^{0} \pi^{0}\right) \eta\right)=(1.6 \pm 0.5) \times 10^{-3}$, this is a relevant approximation to predict the decay rate of this channel.

\subsection{Calculation of the branching ratio $\boldsymbol{B} \boldsymbol{R}\left(\boldsymbol{D}^{+} \rightarrow \boldsymbol{\pi}^{+} \boldsymbol{\eta}\right)$}

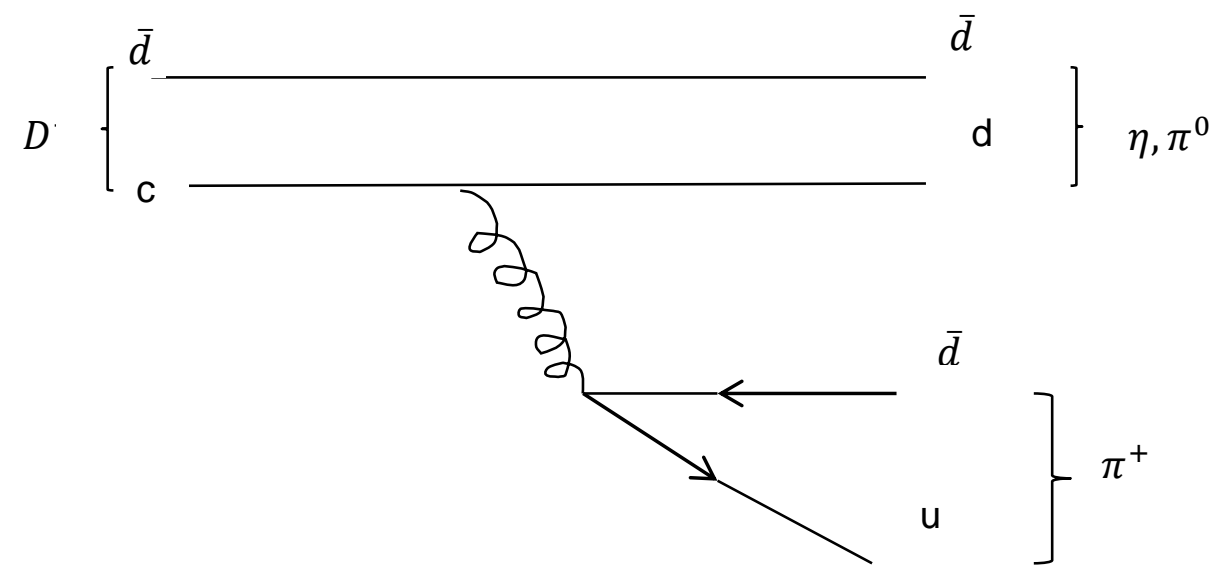

$s / d$

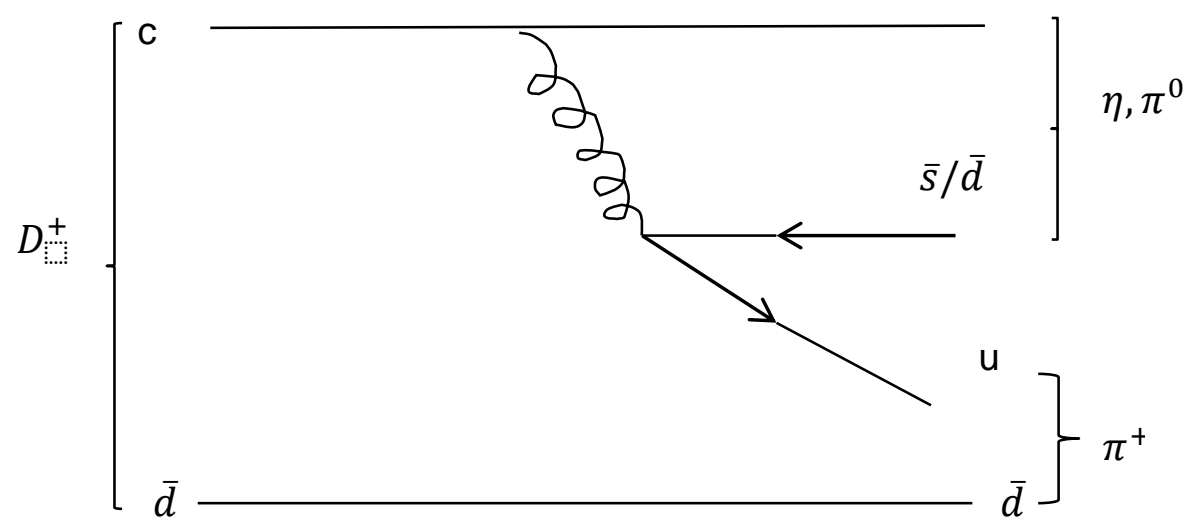

Figure 4. Typical diagram of $D^{+} \rightarrow \pi^{0} \pi^{+} / D^{+} \rightarrow \eta \pi^{+}$for Class. 
First at all, using the factorization, as same as $A\left(D_{S}^{+} \rightarrow \pi^{+} \eta^{\prime}\right)$, we calculate the amplitude of $D^{+} \rightarrow \pi^{+} \eta$ in Figure 4

$$
\begin{aligned}
A\left(D^{+} \rightarrow \pi^{+} \eta\right) & =A_{a}\left(D^{+} \rightarrow \pi^{+} \eta\right)+A_{b}\left(D^{+} \rightarrow \pi^{+} \eta\right)+A_{c}\left(D^{+} \rightarrow \pi^{+} \eta\right) \\
& =\frac{1}{\sqrt{3}} V_{c s} V_{u s} C+\left(-\frac{1}{\sqrt{3}}\right) V_{c d} V_{u d} C+\left(-\frac{1}{\sqrt{3}}\right) V_{c d} V_{u d} T \\
& =\frac{1}{\sqrt{3}} \lambda C+\left(-\frac{1}{\sqrt{3}}\right)(-\lambda) C+\left(-\frac{1}{\sqrt{3}}\right)(-\lambda) T=\frac{2}{\sqrt{3}} \lambda C+\frac{1}{\sqrt{3}} \lambda T
\end{aligned}
$$

Where $V_{c s} \approx 1 ; V_{u d} \approx 1 ; V_{u s}=\lambda ; V_{c d}=-\lambda$

$$
\begin{gathered}
C=a_{2}\left\langle\eta\left|A_{\mu}\right| 0\right\rangle\left\langle\pi^{+}\left|V_{\mu}\right| D^{+}\right\rangle \\
T=a_{1}\left\langle\pi^{+}\left|A_{\mu}\right| 0\right\rangle\left\langle\eta\left|V_{\mu}\right| D^{+}\right\rangle
\end{gathered}
$$

Typical calculation for $\mathrm{C}$ and $\mathrm{T}$ in Eq. $\left(^{*}\right)$, we have:

$$
\begin{gathered}
C=a_{2} i f p_{\eta \mu}\left(p_{D^{+}}+p_{\pi^{+}}\right)^{\mu} F_{0}^{D^{+} \rightarrow \pi^{+}}(0)=a_{2} i f\left(m_{D^{+}}^{2}-m_{\pi^{+}}^{2}\right) F_{0}^{D^{+} \rightarrow \pi^{+}}(0)(16) \\
T=a_{1} i f p_{\pi^{+} \mu}\left(p_{D^{+}}+p_{\eta}\right)^{\mu} F_{0}^{D^{+} \rightarrow \eta}(0)=a_{1} i f\left(m_{D^{+}}^{2}-m_{\eta}^{2}\right) F_{0}^{D^{+} \rightarrow \eta}(0)(17)
\end{gathered}
$$

We obtain the branching ratio of $D^{+} \rightarrow \pi^{+} \eta$

$$
\begin{aligned}
B R\left(D^{+} \rightarrow \pi^{+} \eta\right) & \sim \tau\left(D^{+}\right)\left|A\left(D^{+} \rightarrow \pi^{+} \eta\right)\right|^{2} \\
& =\tau\left(D^{+}\right) \frac{\lambda^{2}}{3} \mid 2 a_{2} i f\left(m_{D^{+}}^{2}-m_{\pi^{+}}^{2}\right) F_{0}^{D^{+} \rightarrow \pi^{+}} \\
& +\left.a_{1} i f\left(m_{D^{+}}^{2}-m_{\eta}^{2}\right) F_{0}^{D^{+} \rightarrow \eta}(0)\right|^{2}
\end{aligned}
$$

From the Figure 4, we compute the branching ratio of $D^{+} \rightarrow \pi^{+} \pi^{0}$

$$
\begin{aligned}
B R\left(D^{+} \rightarrow \pi^{+} \pi^{0}\right) & \sim \tau\left(D^{+}\right)\left|A\left(D^{+} \rightarrow \pi^{+} \pi^{0}\right)\right|^{2} \\
& =\tau\left(D^{+}\right) \frac{\lambda^{2}}{2} \mid a_{2} i f\left(m_{D^{+}}^{2}-m_{\pi^{+}}^{2}\right) F_{0}^{D^{+} \rightarrow \pi^{+}}(0) \\
& +a_{1} \text { if }\left.\left(m_{D^{+}}^{2}-m_{\pi^{0}}^{2}\right) F_{0}^{D^{+} \rightarrow \pi^{0}}(0)\right|^{2}
\end{aligned}
$$

Taking the ratio between the branching ratio of $D^{+} \rightarrow \pi^{+} \eta$ and $D^{+} \rightarrow \pi^{+} \pi^{0}$,

$$
\begin{aligned}
\frac{B R\left(D^{+} \rightarrow \pi^{+} \eta\right)}{B R\left(D^{+} \rightarrow \pi^{+} \pi^{0}\right)} & =\frac{\tau\left(D^{+}\right)\left|A\left(D^{+} \rightarrow \pi^{+} \eta\right)\right|^{2}}{\tau\left(D^{+}\right)\left|A\left(D^{+} \rightarrow \pi^{+} \pi^{0}\right)\right|^{2}} \\
& =\frac{\frac{\lambda^{2}}{3}\left|2 a_{2} i f\left(m_{D^{+}}^{2}-m_{\pi^{+}}^{2}\right) F_{0}^{D^{+} \rightarrow \pi^{+}}(0)+a_{1} i f\left(m_{D^{+}}^{2}-m_{\eta}^{2}\right) F_{0}^{D^{+} \rightarrow \eta}(0)\right|^{2}}{\frac{\lambda^{2}}{2}\left|a_{2} i f\left(m_{D^{+}}^{2}-m_{\pi^{+}}^{2}\right) F_{0}^{D^{+} \rightarrow \pi^{+}}(0)+a_{1} i f\left(m_{D^{+}}^{2}-m_{\pi^{0}}^{2}\right) F_{0}^{D^{+} \rightarrow \pi^{0}}(0)\right|^{2}} \\
= & \frac{2}{3}\left(\frac{2 \frac{a_{2}}{a_{1}}+x_{1}}{\frac{a_{2}}{a_{1}}+x_{2}}\right)^{2}
\end{aligned}
$$

Where $x_{1}=\frac{\left(m_{D^{+}}^{2}-m_{\eta}^{2}\right) F_{0}^{D^{+} \rightarrow \eta}(0)}{\left(m_{D^{+}}^{2}-m_{\pi^{+}}^{2}\right) F_{0}^{D^{+} \rightarrow \pi^{+}}(0)} ; x_{2}=\frac{\left(m_{D^{+}}^{2}-m_{\pi^{0}}^{2}\right) F_{0}^{D^{+} \rightarrow \pi^{0}}(0)}{\left(m_{D^{+}}^{2}-m_{\pi^{+}}^{2}\right) F_{0}^{D^{+} \rightarrow \pi^{+}}(0)}$

Given values as $[3,5]: \mathrm{a}_{2} / \mathrm{a}_{1}=-0.445, B R\left(D^{+} \rightarrow \pi^{+} \pi^{0}\right)=1.19 \times 10^{-3}$, we have:

$B R\left(D^{+} \rightarrow \pi^{+} \eta\right)=0.313 \times 10^{-3}$ vs $B R_{\text {exp }}\left(D^{+} \rightarrow \pi^{+} \eta\right)=(3.53 \pm 0.21) \times 10^{-3}$.

In three above applications of $\mathrm{D}$ mesons, we end this section with one remark: Factorization of hadronic matrix elements of four-quark operators into two matrix elements of color-singlet currents 
implies that only those non-perturbative forces that act between quarks and antiquarks are taken into account. In this case, we have not considered the remaining interaction, in particular, the gluon exchange between two quarks or two antiquarks. That is the reason why we see a small difference between the theoretical and experimental result in $\mathrm{D}_{\mathrm{s}}^{+}, \mathrm{D}^{0}$ (Class I, II) and a larger difference between the theoretical and experimental result in $\mathrm{D}^{+}$(Class III).

\section{Conclusion}

Via factorization, we compute thechannels $B R\left(D^{+} \rightarrow \pi^{+} \eta\right)=0.313 \times 10^{-3}, B R\left(D_{s}^{+} \rightarrow \pi^{+} \eta^{\prime}\right)=$ $2.31 \times 10^{-2}, \quad B R\left(D^{0} \rightarrow \bar{K}^{0 *}(892)\left(\rightarrow K_{S}{ }^{0} \pi^{0}\right) \eta\right)=4.4 \times 10^{-3}$. Read-backing with experimental values [5], the resultscan be acceptable.Also, factorization should be progressed in the gluon interaction between two quarks or two antiquarks.

Therefore, factorization method can be practical for the new channels in the future to estimate the decay rate of charmed mesons at low energy QCD and in general at low energy QCD, at some physical regions we do not understand about their theories.

\section{Acknowledgments}

We thank Dr. Tran Minh Hieu for clarifying correspondence.

\section{References}

[1] N.T. Huong, E. Kou and B. Viaud, Novel approach to measure the leptonic $\eta\left({ }^{\prime}\right) \rightarrow \mu^{+} \mu^{-}$decays via charmed meson decays, Phys.Rev. D 94, 054040 (2016).

[2] M. Artuso, B. Meadows and Alexey A. Petrov, Charm Meson Decays, Annual Review of Nuclear and Particle Science, Vol. 58: 249-291 (November 2008).

[3] Andrzej J. Buras, Weak Hamiltonian- CP Violation and Rare Decays, arXiv: 9806471[hep-ph].

[4] M.K. Gaillard and B.W. Lee, $\Delta \mathrm{I}=12$ Rule for Nonleptonic Decays in Asymptotically Free Field Theories, Phys. Rev. Lett. 33, 108 (1974).

[5] G. Altarelli and L. Maiani, Octet Enhancement of Nonleptonic Weak Interactions in Asymptotically Free Gauge Theories, Phys. Lett. B 52, 351 (1974).

[6] M. Neubert, B. Stech, Non-Leptonic Weak Decays of B Mesons, Adv. Ser. Direct. High Energy Phys.15:294344,1998, arXiv: 9705292[hep-ph]

[7] K. A. Olive et al. (Particle Data Group), Review of Particle Physics, Chin. Phys. C, 38, 090001 (2014).

[8] T.N. Pham, $\eta-\eta$ ' mixing, Phys. Rev. D 92, 054021 (2015).

[9] A. Bramon, R. Escribano and M. D. Scadron, The eta - eta-prime mixing angle revisited, Eur.Phys.J.C7:271278,1999 .

\section{Appendix}

Definition for the weak decay form factors[3]: It parametrize the hadronic matrix elements of flavor- changing vector and axial currents between meson states.

For the transition between two pseudoscalar mesons, $\mathrm{P}_{1}(\mathrm{p}) \rightarrow \mathrm{P}_{2}(\mathrm{p}$ '), we define:

$$
\left\langle P_{2}\left(p^{\prime}\right)\left|V_{\mu}\right| P_{1}(p)\right\rangle=\left(\left(p+p^{\prime}\right)_{\mu}-\frac{m_{1}^{2}-m_{2}^{2}}{q^{2}} q_{\mu}\right) F_{1}\left(q^{2}\right)+\frac{m_{1}^{2}-m_{2}^{2}}{q^{2}} q_{\mu} F_{0}\left(q^{2}\right)
$$

Moreover, in order for the poles at $\mathrm{q}^{2}=0$ to cancel, we must impose the conditions $\mathrm{F}_{1}(0)=\mathrm{F}_{0}(0)$. 